\title{
Instability of sloshing motion in a vessel undergoing pivoted oscillations
}

\author{
by M.R. Turner ${ }^{1}$, H. Alemi Ardakani \& T.J. Bridges \\ Department of Mathematics, University of Surrey, \\ Guildford, Surrey GU2 7XH, England
}

- Abstract -

Suspending a rectangular vessel partially filled with an inviscid fluid from a single rigid pivoting rod produces an interesting physical model for investigating the dynamic coupling between the fluid and vessel motion. The fluid motion is governed by the Euler equations relative to the moving frame of the vessel, and the vessel motion is given by a modified forced pendulum equation. The fully nonlinear, two-dimensional, equations of motion are derived and linearized for small-amplitude vessel and free-surface motions, and the natural frequencies of the system analysed. It is found that the linear problem exhibits an unstable solution if the rod length is shorter than a critical length which depends on the length of the vessel, the fluid height and the ratio of the fluid and vessel masses. In addition, we identify the existence of $1: 1$ resonances in the system where the symmetric sloshing modes oscillate with the same frequency as the coupled fluid/vessel motion. The implications of instability and resonance on the nonlinear problem are also briefly discussed.

Keywords: sloshing, pivoted, oscillations, instability, homoclinic

\section{Introduction}

The movement of a vessel partially filled with a fluid can cause the fluid motion to undergo extremely complex motions. Moreover, the fluid motion, comprising waves sloshing back and forth along the fluid free-surface, produce forces and moments on the vessel, which if the vessel is free to move, can cause unintended vessel motion which could be stabilizing or destabilizing. A simple example of such unintended motion can be found in the article 'walking with coffee' which examines the spilling of coffee while walking (Mayer and Krechetnikov, 2012). A more dramatic example of a destabilizing fluid motion is the dynamics of trapped seawater on the deck of Alaskan king crab boats. They

\footnotetext{
${ }^{1}$ Corresponding Author: m.turner@surrey.ac.uk
} 
have been observed to capsize when the trapped water sloshes backwards and forwards creating unintended moments enhancing the roll motion of the boat (Dillingham, 1981; Caglayan and Storch, 1982; Adee and Caglayan, 1982). Therefore, the ability to identify destabilizing motions in coupled fluid-vessel interactions is of great practical importance. Examples where this coupling is important are terrestrial and maritime fluid transportation, space transport, fuel tanks under earthquake excitement and industrial applications such as tuned liquid dampers (TLDs) (Ikeda and Nakagawa, 1997; Frandsen, 2005).

Studying the motion of a fluid in a stationary or forced vessel is already very complicated both experimentally and theoretically. The works by Moiseyev and Rumyantsev (1968), Ibrahim (2005) and Faltinsen and Timokha (2009), and the references herein, highlight the problems in these areas. The problem of coupled dynamics adds an additional layer of complexity to this problem because it allows for the potential enhancement or destabilization of fluid dynamics due to the motion of the vessel.

The configuration of interest in this paper is shown in Figure 1. The vessel, with

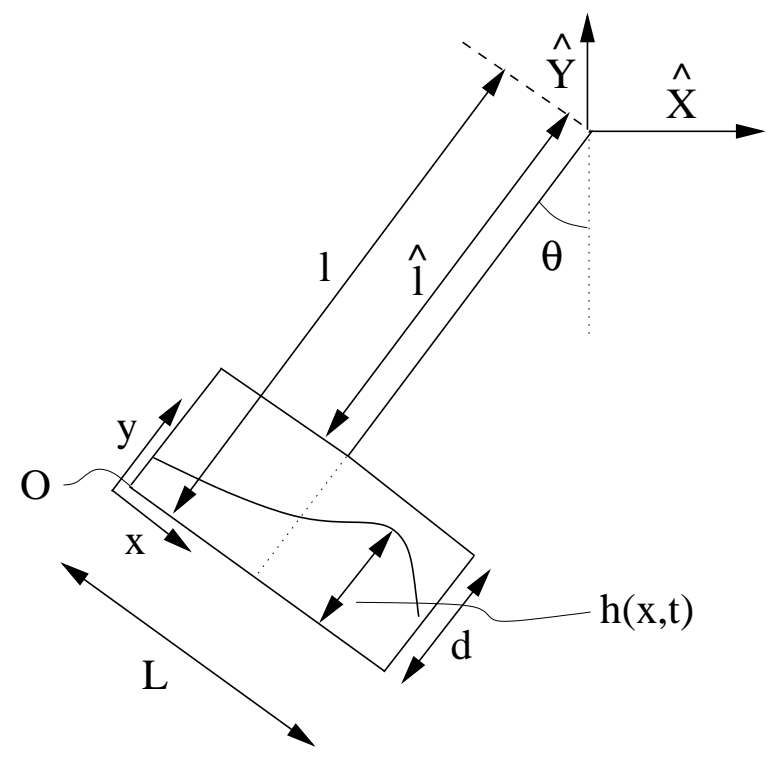

Figure 1: Pendulum vessel experiment under consideration.

rectangular cross section of length $L$ and height $d$, is suspended by a rigid rod of length $\widehat{l}$ which is attached to the top of the vessel and is free to rotate in the vertical plane such that the rod makes an angle $\theta$ with the downward vertical. The values of $\widehat{l}$ and $d$ are only important in the combination $l=\widehat{l}+d$, which is the perpendicular distance from the pivot point to the base of the vessel. The vessel is partially filled with an inviscid, incompressible, constant density fluid of mass $m_{f}$ and density $\rho$. When the vessel is in motion the free surface of the fluid is given by $y=h(x, t)$, with mean depth $h_{0}$, where $\mathbf{x}=(x, y)$ is a coordinate system fixed to the moving vessel. The base of the vessel is at $y=0$. We also define $\widehat{\mathbf{X}}=(\widehat{X}, \widehat{Y})$ to be a planar fixed coordinate system with origin at the point at which the vessel pivots. The fluid mass $m_{f}=\int_{0}^{L} \rho h(x, t) d x$ is independent of time. This pivoting TLD setup is of interest to engineers, because it is a good mechanism for suppressing torsional vibrations on bridges caused by aerodynamic effects (Xue et al., 2000; Chen et al., 2008), which are a danger to high sided vehicles (Chen and Cai, 2004). 
These studies include experiments as well as linear and nonlinear simulations, where the shallow water model is assumed for the fluid motion.

This configuration is the simplest coupling between fluid and vessel motion that allows rotation. The TLD configuration is simpler but only allows for translation of the vessel. This pendulum-slosh model was one of the first coupled models to be studied (Moiseev, 1953; Abramson et al., 1961; Moiseyev and Rumyantsev, 1968). Indeed, the linear equations of motion were first derived in Moiseyev and Rumyantsev (1968) by considering the added mass coefficients for the fluid and a Lagrangian construction for the two-dimensional vessel equation. They then went on to derive the characteristic relation for linear perturbations in terms of a general vessel geometry. In this paper we present three new results for this linear coupled problem: firstly, we give a new independent derivation of the governing equations and characteristic equation for the natural frequencies, confirming the result in Moiseyev and Rumyantsev (1968); secondly, we have discovered a new instability of this coupled system; and thirdly, we have discovered a $1: 1$ resonance in the system.

The instability range is surprising because it always occurs with the pivot point above the centre of mass of the quiescent fluid. If the fluid was a rigid body of length $L$ and height $h_{0}$ and the pivot point was on the vertical centreline, the configuration would be unstable if and only if the pivot point was below the centroid,

$$
l<\frac{1}{2} h_{0} .
$$

By replacing the rigid body with a fluid in the interior, the instability can arise with the pivot point above the point $y=\frac{1}{2} h_{0}$. We have discovered the remarkable and exact formula

$$
(1+R) l<\frac{1}{2} h_{0}+\frac{1}{12} \frac{L^{2}}{h_{0}},
$$

for the instability threshold, where

$$
R=\frac{m_{v}}{m_{f}}
$$

and $m_{v}$ is the mass of the dry vessel. For example the instability threshold can be even greater than $h_{0}$ (above the still water level), depending on the values of $R, h_{0}$ and $L$. The depth ratio $\frac{h_{0}}{L}$ of the fluid plays a key role. The effect of this instability on mechanical structures, such as the TLD for torsional bridge oscillations, would be catastrophic, leading to large unstable oscillations, which could ultimately cause structural damage.

This configuration is to contrasted with Cooker's experiment (Cooker, 1994), which is also pendular, but with two suspension points so the base of the vessel always remains horizontal. In this configuration the trivial solution is never unstable. It has however many other features of interest. It has been studied experimentally by Cooker (1994) and Weidman (1994, 2005) and Herczyński and Weidman (2012) with the latter works considering multi-compartmental vessels, different vessel geometries and the special case where the string length of the pivot point tends to infinity. There has been extensive theoretical study of the Cooker configuration as well. Cooker (1994) compared the results of his experiments to a theoretical model in which the fluid was assumed to be shallow, and the vessel motion was modelled using a linear pendulum model. Various extensions of this problem have been considered such as introducing a finite depth fluid (Yu, 2010), 
having a nonlinear shallow water fluid (Alemi Ardakani and Bridges, 2010) and including the fully nonlinear vessel motion (Alemi Ardakani et al., 2012a). Alemi Ardakani et al. (2012a,b) also highlighted the existence of an internal $1: 1$ resonance in the Cooker experiment, where the symmetric sloshing modes are dynamically coupled to the antisymmetric sloshing modes, and hence the vessel motion. Such resonances are pathways for energy exchange within fluid modes and Turner and Bridges (2013) showed that for a rectangular vessel, there is a single fluid height where there exists a heteroclinic orbit between the purely symmetric and purely anti-symmetric modes. Solutions close to this heteroclinic orbit can manifest themselves in an experiment by having an oscillating vessel slowly coming to rest as the energy is transferred from the vessel to the symmetric modes or a stationary vessel containing a symmetric sloshing mode starting to oscillate as the energy is transferred to the anti-symmetric modes, and hence the vessel.

The main new result in the pendular configuration in Figure 1 is the emergence of instability in a region where one would normally expect stability. Since the system is conservative the instability arises through a pair of purely imaginary eigenvalues meeting at the origin and becoming a real hyperbolic pair as shown schematically in Figure 2. One of the interesting outcomes of this instability is that the behaviour in the weakly

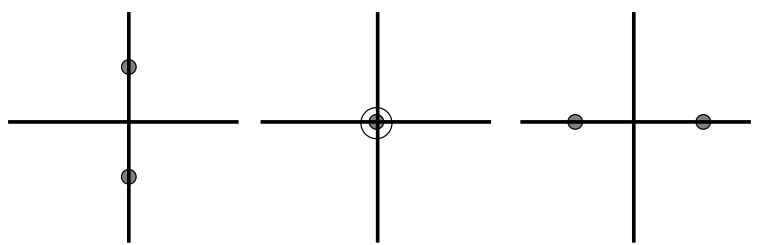

Figure 2: Schematic of the transition from stability to instability.

nonlinear problem is well-known in the Hamiltonian systems literature (Meyer and Hall, 1992; Arnold et al., 1993; Bridges, 2012). In the weakly nonlinear problem a homoclinic orbit emerges, which may lead to complex dynamics for the nonlinear system near the instability transition. The implications for the present configuration are discussed in $\S 4$.

The structure of the paper is as follows. The formulation of the governing nonlinear equations for the coupled pendulum problem are given in $\S 2$, while $\S 3$ analyses the linearized equations and we derive the characteristic function for the natural frequencies in $§ 3.1$. Section 4 discusses the implications of the nonlinear problem on the instability: a homoclinic orbit is generated which could produce complex fluid motion near the instability threshold. Conclusions and further discussions can be found in $\S 5$.

\section{Formulation of governing equations}

The configuration of interest is shown in Figure 1. The two Cartesian coordinate systems are related via

$$
\widehat{\mathbf{X}}=\mathbf{Q}(t)(\mathbf{x}+\mathbf{d}),
$$

where

$$
\mathbf{Q}(t)=\left(\begin{array}{cc}
\cos \theta & -\sin \theta \\
\sin \theta & \cos \theta
\end{array}\right)
$$


is a rotation tensor in $\mathbb{R}^{2}$ and

$$
\mathbf{d}=\left(\begin{array}{c}
d_{1} \\
d_{2}
\end{array}\right)=\left(\begin{array}{c}
-L / 2 \\
-l
\end{array}\right)
$$

is the displacement of the axis of rotation from the origin of the body frame. We have assumed for simplicity that the rigid rod is fixed along the centre line of the vessel (i.e. $\left.d_{1}=-L / 2\right)$, but we leave the quantities $d_{1}$ and $d_{2}$ in the analysis to simplify the algebra.

If we define a velocity vector $\mathbf{u}=(u, v)$ to be the Eulerian velocity vector in the body coordinate system then the Euler equations of motion are

$$
\begin{aligned}
& \frac{D u}{D t}+\frac{1}{\rho} \frac{\partial p}{\partial x}=-g \sin \theta+2 \dot{\theta} v+\ddot{\theta}\left(y+d_{2}\right)+\dot{\theta}^{2}\left(x+d_{1}\right), \\
& \frac{D v}{D t}+\frac{1}{\rho} \frac{\partial p}{\partial y}=-g \cos \theta-2 \dot{\theta} u-\ddot{\theta}\left(x+d_{1}\right)+\dot{\theta}^{2}\left(y+d_{2}\right),
\end{aligned}
$$

where $g$ denotes the constant acceleration due to gravity and the dots denote differentiation with respect to $t$. The Euler equations can be derived directly from equation (2.4) of Alemi Ardakani and Bridges (2011), which gives the 3D form of the Euler equations in a rotating frame, if we set $\boldsymbol{\Omega}=(0,0, \dot{\theta}), \mathbf{q}=\mathbf{0}$ in their notation, and consider gravity acting in the negative $y$-direction.

As the fluid is assumed to be incompressible we also have the continuity equation

$$
\frac{\partial u}{\partial x}+\frac{\partial v}{\partial y}=0
$$

while the boundary conditions are the no penetration conditions on the rigid vessel walls, and the dynamic and kinematic conditions on the free-surface $y=h(x, t)$,

$$
\begin{aligned}
v=0 & \text { on } \quad y=0, \\
u=0 & \text { on } \quad x=0, L, \\
p=0 & \text { on } \quad y=h(x, t), \\
h_{t}+u h_{x}=v & \text { on } \quad y=h(x, t) .
\end{aligned}
$$

The motion of the fluid within the vessel is dynamically coupled to the motion of the vessel itself via a pendulum equation which can be derived by considering the Lagrangian formulation for the vessel. The Lagrangian for the vessel motion for $2 \mathrm{D}$ rigid body motion is given by equation (6.2.7) of Alemi Ardakani (2010) (with $q_{1}=q_{2}=0$ ) as

$$
\mathcal{L}=\int_{t_{1}}^{t_{2}} \mathscr{L} d t
$$

where

$$
\begin{aligned}
\mathscr{L}= & \int_{0}^{L} \int_{0}^{h}\left[\frac{1}{2}\left(u^{2}+v^{2}\right)-\dot{\theta} u\left(y+d_{2}\right)+\dot{\theta} v\left(x+d_{1}\right)+\frac{1}{2} \dot{\theta}^{2}\left(\left(x+d_{1}\right)^{2}+\left(y+d_{2}\right)^{2}\right)\right. \\
& \left.-g\left(\sin \theta\left(x+d_{1}\right)+\cos \theta\left(y+d_{2}\right)\right)\right] \rho d y d x+\frac{1}{2} m_{v}\left[\left(x_{v}+d_{1}\right)^{2}+\left(y_{v}+d_{2}\right)^{2}\right] \dot{\theta}^{2} \\
& -m_{v} g\left[\left(x_{v}+d_{1}\right) \sin \theta+\left(y_{v}+d_{2}\right) \cos \theta\right] .
\end{aligned}
$$


Here $\left(x_{v}, y_{v}\right)$ is the position of the centre of mass of the vessel in the body frame coordinates, hence $x_{v}$ and $y_{v}$ are constants for a fixed vessel geometry.

The vessel equation can then be found by considering the Euler-Lagrange equation $\frac{d}{d t}\left(\frac{\partial \mathscr{L}}{\partial \dot{\theta}}\right)-\frac{\partial \mathscr{L}}{\partial \theta}=0$ for $\theta$ which gives

$$
\begin{aligned}
& \left(m_{v}\left[\left(x_{v}+d_{1}\right)^{2}+\left(y_{v}+d_{2}\right)^{2}\right]+\int_{0}^{L} \int_{0}^{h}\left[\left(x+d_{1}\right)^{2}+\left(y+d_{2}\right)^{2}\right] \rho d y d x\right) \ddot{\theta} \\
& +\dot{\theta} \frac{d}{d t} \int_{0}^{L} \int_{0}^{h}\left[\left(x+d_{1}\right)^{2}+\left(y+d_{2}\right)^{2}\right] \rho d y d x+m_{v} g\left[\left(x_{v}+d_{1}\right) \cos \theta-\left(y_{v}+d_{2}\right) \sin \theta\right] \\
& +g \int_{0}^{L} \int_{0}^{h}\left[\cos \theta\left(x+d_{1}\right)-\sin \theta\left(y+d_{2}\right)\right] \rho d y d x \\
& =\frac{d}{d t}\left[\int_{0}^{L} \int_{0}^{h}\left[u\left(y+d_{2}\right)-v\left(x+d_{1}\right)\right] \rho d y d x\right] .
\end{aligned}
$$

Due to the rotation of the vessel the flow is not irrotational, as it is in the Cooker or TLD experimental setup (Cooker, 1994; Frandsen, 2005; Alemi Ardakani et al., 2012a) where the base of the vessel remains horizontal during motion. The vorticity for this flow $\omega=\frac{\partial v}{\partial x}-\frac{\partial u}{\partial y}$ satisfies the equation

$$
\frac{D \omega}{D t}=\frac{\partial}{\partial x}\left(\frac{D v}{D t}\right)-\frac{\partial}{\partial y}\left(\frac{D u}{D t}\right)-\left(\frac{\partial u}{\partial x}+\frac{\partial v}{\partial y}\right) \omega=-2 \ddot{\theta}
$$

which leads to the conclusion that $\omega$ is constant in space and $\omega=-2 \dot{\theta}$ everywhere. Therefore, in order to analyse this problem using methods developed for the irrotational Cooker experiment, we split the flow into a rotational part and an irrotational part and introduce a velocity potential $\phi(x, y, t)$ for the irrotational part. Thus we let

$$
u=\frac{\partial \phi}{\partial x}, \quad \text { and } \quad v=\frac{\partial \phi}{\partial y}-2 \dot{\theta}\left(x+d_{1}\right)
$$

Obviously, we could define $\phi$ in different ways, and the reason for this choice for $\phi$ is discussed below. The continuity equation then leads to Laplace's equation for $\phi$

$$
\phi_{x x}+\phi_{y y}=0 \quad \text { in } \quad 0 \leq x \leq L, \quad 0 \leq y \leq h(x, t),
$$

and integrating (2.1) and (2.2) with respect to $x$ and $y$ respectively leads to Bernoulli's equation for the pressure

$$
\begin{aligned}
& \frac{p}{\rho}+\phi_{t}+\frac{1}{2}\left(\phi_{x}^{2}+\phi_{y}^{2}\right)-2 \dot{\theta}\left(x+d_{1}\right) \phi_{y}-\ddot{\theta}\left(y+d_{2}\right)\left(x+d_{1}\right) \\
& -\frac{1}{2} \dot{\theta}^{2}\left[-3\left(x+d_{1}\right)^{2}+\left(y+d_{2}\right)^{2}\right]+g(x \sin \theta+y \cos \theta)=B e(t),
\end{aligned}
$$

where $B e(t)$ is the Bernoulli function which we absorb into $\phi(x, y, t)$. Therefore the dynamic boundary condition (2.5) becomes

$$
\begin{aligned}
& \phi_{t}+\frac{1}{2}\left(\phi_{x}^{2}+\phi_{y}^{2}\right)-2 \dot{\theta}\left(x+d_{1}\right) \phi_{y}-\ddot{\theta}\left(h+d_{2}\right)\left(x+d_{1}\right) \\
& -\frac{1}{2} \dot{\theta}^{2}\left[-3\left(x+d_{1}\right)^{2}+\left(h+d_{2}\right)^{2}\right]+g(x \sin \theta+h \cos \theta)=0, \quad \text { on } y=h(x, t) .
\end{aligned}
$$


In terms of the velocity potential $\phi(x, y, t)$, the other boundary conditions become

$$
\begin{aligned}
& \frac{\partial \phi}{\partial x}=0, \quad \text { on } \quad x=0, L \\
& \frac{\partial \phi}{\partial y}=2 \dot{\theta}\left(x+d_{1}\right), \quad \text { on } \quad y=0
\end{aligned}
$$

and the kinematic free-surface condition becomes

$$
h_{t}+\phi_{x} h_{x}=\phi_{y}-2 \dot{\theta}\left(x+d_{1}\right) \quad \text { on } \quad y=h(x, t) .
$$

The motivation for choosing the form for $\phi(x, y, t)$ in $(2.9)$ is so that the side wall boundary conditions (2.12) are homogeneous and thus the solution can be expressed as a sum of cosine modes which automatically satisfy this condition, see $\S 3$.

Substituting (2.9) into (2.8) gives the form of the vessel equation as

$$
\begin{aligned}
& \left(m_{v}\left[\left(x_{v}+d_{1}\right)^{2}+\left(y_{v}+d_{2}\right)^{2}\right]+\int_{0}^{L} \int_{0}^{h}\left[\left(x+d_{1}\right)^{2}+\left(y+d_{2}\right)^{2}\right] \rho d y d x\right) \ddot{\theta} \\
& +\dot{\theta} \frac{d}{d t} \int_{0}^{L} \int_{0}^{h}\left[\left(x+d_{1}\right)^{2}+\left(y+d_{2}\right)^{2}\right] \rho d y d x+m_{v} g\left(\left(x_{v}+d_{1}\right) \cos \theta-\left(y_{v}+d_{2}\right) \sin \theta\right) \\
& +g \int_{0}^{L} \int_{0}^{h}\left[\cos \theta\left(x+d_{1}\right)-\sin \theta\left(y+d_{2}\right)\right] \rho d y d x \\
& =\frac{d}{d t}\left[\int_{0}^{L} \int_{0}^{h}\left[\phi_{x}\left(y+d_{2}\right)-\left(\phi_{y}-2 \dot{\theta}\left(x+d_{1}\right)\right)\left(x+d_{1}\right)\right] \rho d y d x\right]
\end{aligned}
$$

The Lagrangian in (2.7) can be compared to that given in $\S 5-4$ of Moiseyev and Rumyantsev (1968), see Appendix A for this comparison. However, this Lagrangian only contains information about the coupled vessel motion, it doesn't contain the fluid motion. The full Lagrangian for the coupled system which does includes the fluid equations is

$$
\begin{aligned}
\mathscr{L}= & \int_{0}^{L} \int_{0}^{h} \rho\left[\phi_{t}+\frac{1}{2}\left(\phi_{x}^{2}+\phi_{y}^{2}\right)-2 \dot{\theta}\left(x+d_{1}\right) \phi_{y}-\ddot{\theta}\left(h+d_{2}\right)\left(x+d_{1}\right)\right. \\
& \left.-\frac{1}{2} \dot{\theta}^{2}\left[-3\left(x+d_{1}\right)^{2}+\left(h+d_{2}\right)^{2}\right]+g\left(\left(x+d_{1}\right) \sin \theta+\left(y+d_{2}\right) \cos \theta\right)\right] d y d x \\
& -\frac{1}{2} m_{v}\left[\left(x_{v}+d_{1}\right)^{2}+\left(y_{v}+d_{2}\right)^{2}\right] \dot{\theta}^{2}+m_{v} g\left[\left(x_{v}+d_{1}\right) \sin \theta+\left(y_{v}+d_{2}\right) \cos \theta\right] .
\end{aligned}
$$

In the next section we investigate linear perturbations of the governing equations about a quiescent state.

\section{Linear Analysis}

The nonlinear governing equations for the coupled problem are (2.10)-(2.14) for the fluid motion and (2.15) for the vessel motion. To find the characteristic function for the small amplitude solutions we linearize these equations about a state of quiescent fluid where $\phi=-g h_{0} t, h=h_{0}, \theta=0$ where $h_{0}$ is a constant. The perturbation is assumed to be 
harmonic so we write

$$
\begin{aligned}
\phi(x, y, t) & =-g h_{0} t+\frac{1}{2}\left[\widehat{\phi}(x, y) e^{\mathrm{i} \omega t}+\mathrm{c.c}\right], \\
h(x, t) & =h_{0}+\frac{1}{2}\left[\mathrm{i} \widehat{h}(x) e^{\mathrm{i} \omega t}+\text { c.c }\right] \\
\theta(t) & =\frac{1}{2} \mathrm{i} \hat{\theta} e^{\mathrm{i} \omega t}+\text { c.c }
\end{aligned}
$$

where $\omega=\Omega_{r}+\mathrm{i} \Omega_{i}$ is the complex angular frequency, c.c denotes the complex conjugate and $\widehat{\phi}$ and $\widehat{h}$ are complex functions with $\widehat{\theta}$ real. Note it is assumed that $|\widehat{\phi}|,|\widehat{h}|,|\widehat{\theta}| \ll 1$. Also at this stage we make the conscious decision to stipulate the position of the centre of mass of the vessel. To make the algebra more convenient we set the centre of mass of the dry vessel to be at $\left(x_{v}, y_{v}\right)=\left(-d_{1}, 0\right)$, i.e. at the centre of the base of the vessel. We expect small deviations from this position only to make a small difference to the results presented here, and this position would be obtainable for a physical vessel, by adding additional mass to the bottom.

Substituting these expressions into the governing equations $(2.10,2.14,2.11,2.12,2.13)$ respectively, and retaining only linear terms leads to

$$
\begin{aligned}
\widehat{\phi}_{x x}+\widehat{\phi}_{y y} & =0 \text { in } 0 \leq x \leq L, \quad 0 \leq y \leq h_{0}, \\
-\omega \hat{h} & =\widehat{\phi}_{y}+2 \omega \widehat{\theta}\left(x+d_{1}\right) \quad \text { on } y=h_{0}, \\
\omega \widehat{\phi}+\omega^{2} \widehat{\theta}\left(h_{0}+d_{2}\right)\left(x+d_{1}\right)+g(x \widehat{\theta}+\widehat{h}) & =0 \text { on } y=h_{0}, \\
\frac{\partial \widehat{\phi}}{\partial x} & =0 \text { on } x=0, L, \\
\frac{\partial \widehat{\phi}}{\partial y} & =-2 \omega \widehat{\theta}\left(x+d_{1}\right) \quad \text { on } y=0,
\end{aligned}
$$

and from (2.15) we derive

$$
\begin{aligned}
& -\omega^{2}\left(\left(m_{v}+m_{f}\right) d_{2}^{2}-\frac{L^{2} m_{f}}{12}+h_{0} m_{f} d_{2}+\frac{h_{0}^{2} m_{f}}{3}\right) \widehat{\theta}-g\left(\left(m_{v}+m_{f}\right) d_{2}+\frac{m_{f} h_{0}}{2}\right) \widehat{\theta} \\
& -\left.\frac{g \rho}{\omega} \int_{0}^{L}\left(x+d_{1}\right) \widehat{\phi}_{y}\right|_{y=h_{0}} d x-\frac{g \rho L^{3}}{6} \widehat{\theta} \\
& =\omega \int_{0}^{L} \int_{0}^{h_{0}}\left[\widehat{\phi}_{x}\left(y+d_{2}\right)-\widehat{\phi}_{y}\left(x+d_{1}\right)\right] \rho d y d x
\end{aligned}
$$

where we have used (3.5) to eliminate $\widehat{h}$ under the single integral sign. We can also eliminate $\widehat{h}$ from (3.5) and (3.6) giving a single free surface condition in terms of $\widehat{\phi}$ and $\widehat{\theta}$

$$
\omega^{2} \widehat{\phi}-g \widehat{\phi}_{y}=g \omega \widehat{\theta}\left(x+d_{1}\right)+g \omega \widehat{\theta} d_{1}-\omega^{3} \widehat{\theta}\left(h_{0}+d_{2}\right)\left(x+d_{1}\right) .
$$

The RHS of (3.10) can be written as a cosine expansion by noting that

$$
x+d_{1}=x-\frac{L}{2}=\sum_{n=0}^{\infty} p_{n} \cos \left(\alpha_{n} x\right), \quad p_{n}=-\frac{4}{L \alpha_{n}^{2}}, \quad \text { for } \quad 0 \leq x \leq L
$$


where $\alpha_{n}=(2 n+1) \pi / L$ represents the wavenumbers for the anti-symmetric sloshing modes, and we have utilized the fact that the rigid rod is fixed to the centre of the vessel. Thus (3.4) and (3.7)-(3.10) form a boundary value problem for $\widehat{\phi}$ and $\widehat{\theta}$.

The general solution to Laplace's equation which satisfies the side wall boundary conditions (3.7) is

$$
\begin{aligned}
\widehat{\phi}=A+B y & +\sum_{n=1}^{\infty}\left(b_{n} \cosh \beta_{n} y+c_{n} \sinh \beta_{n} y\right) \cos \beta_{n} x \\
& +\sum_{n=0}^{\infty}\left(a_{n} \cosh \alpha_{n} y+d_{n} \sinh \alpha_{n} y\right) \cos \alpha_{n} x,
\end{aligned}
$$

where $\beta_{n}=2 n \pi / L$ corresponds to the symmetric sloshing modes and $A, B, a_{n}, b_{n}, c_{n}$ and $d_{n}$ are constants to be determined.

Satisfying the bottom boundary condition (3.8) leads to

$$
B=0, \quad c_{n}=0, \quad d_{n}=-\frac{2 \widehat{\theta} \omega p_{n}}{\alpha_{n}},
$$

using (3.11), thus

$$
\widehat{\phi}=A+\sum_{n=1}^{\infty} b_{n} \cosh \beta_{n} y \cos \beta_{n} x+\sum_{n=0}^{\infty}\left(a_{n} \cosh \alpha_{n} y-\frac{2 \widehat{\theta} \omega p_{n}}{\alpha_{n}} \sinh \alpha_{n} y\right) \cos \alpha_{n} x .
$$

Substituting this expression into the surface boundary condition and equating coefficients of the cosine terms leads to

$$
\begin{aligned}
b_{n}\left[\frac{\omega^{2}}{g}-\beta_{n} \widehat{T}_{n}\right] & =0 \\
A & =\frac{g d_{1} \widehat{\theta}}{\omega}, \\
a_{n} & =\frac{p_{n} \omega \widehat{\theta}}{C_{n}\left(\omega^{2}-g \alpha_{n} T_{n}\right)}\left[\frac{2}{\alpha_{n}} C_{n}\left(\omega^{2} T_{n}-g \alpha_{n}\right)+g-\omega^{2}\left(h_{0}+d_{2}\right)\right],
\end{aligned}
$$

where $C_{n}=\cosh \alpha_{n} h_{0}, T_{n}=\tanh \alpha_{n} h_{0}$ and $\widehat{T}_{n}=\tanh \beta_{n} h_{0}$. The first of these three conditions shows that if $\omega^{2}=\omega_{n}^{2}=g \beta_{n} \widehat{T}_{n}$ then a solution exists with $b_{n} \neq 0$. If the same value of $\omega$ also leads to the coefficients of the $\cos \alpha_{n} x$ terms being non-zero then there exists an internal 1:1 resonance in this system. Such a resonance is a pathway for energy transfer between the symmetric and anti-symmetric modes which in turn leads to complex vessel motions (Alemi Ardakani et al., 2012a; Turner and Bridges, 2013).

Substituting $a_{n}$ into $\widehat{\phi}$ and using (3.5) gives

$$
\begin{aligned}
\widehat{h}= & \sum_{n=1}^{\infty} \frac{p_{n} \widehat{\theta}}{\omega^{2}-g \alpha_{n} T_{n}}\left[\frac{2 \omega^{2}}{C_{n}}-T_{n} \alpha_{n}\left(g-\omega^{2} H_{0}\right)\right] \cos \alpha_{n} x-2 \widehat{\theta}\left(x+d_{1}\right), \\
= & \sum_{n=1}^{\infty} \frac{\widehat{\theta} p_{n}}{\left(W_{1}-g \alpha_{n} T_{n}\right)^{2}+W_{2}^{2}}\left[\left(W_{1}-g \alpha_{n} T_{n}\right)\left(\frac{2 W_{1}}{C_{n}}-T_{n} \alpha_{n}\left(g-W_{1} H_{0}\right)\right)\right. \\
& \left.+W_{2}^{2}\left(\frac{2}{C_{n}}+T_{n} \alpha_{n} H_{0}\right)\right] \cos \alpha_{n} x \\
+ & \mathrm{i} \sum_{n=1}^{\infty} \frac{\widehat{\theta} W_{2} T_{n} \alpha_{n} g p_{n}}{\left(W_{1}-g \alpha_{n} T_{n}\right)^{2}+W_{2}^{2}}\left[1-\frac{2}{C_{n}}-T_{n} \alpha_{n} H_{0}\right] \cos \alpha_{n} x-2 \widehat{\theta}\left(x+d_{1}\right),
\end{aligned}
$$


while on the free surface

$$
\begin{aligned}
\widehat{\phi}= & A+\sum_{n=1}^{\infty} b_{n} \cosh \beta_{n} h_{0} \cos \beta_{n} x+\sum_{n=0}^{\infty} \frac{\omega \widehat{\theta} p_{n}}{\omega^{2}-g \alpha_{n} T_{n}}\left[g-\frac{2 g}{C_{n}}-\omega^{2} H_{0}\right] \cos \alpha_{n} x . \\
= & A+\sum_{n=1}^{\infty} b_{n} \cosh \beta_{n} h_{0} \cos \beta_{n} x+\sum_{n=1}^{\infty} \frac{\widehat{\theta} p_{n} \Omega_{r}}{\left(W_{1}-g \alpha_{n} T_{n}\right)^{2}+W_{2}^{2}} \\
& {\left[\left(W_{1}+2 \Omega_{i}^{2}-g \alpha_{n} T_{n}\right)\left(g-\frac{2 g}{C_{n}}-\Omega_{r}^{2} H_{0}\right)-H_{0} \Omega_{i}^{2}\left(W_{1}+2 \Omega_{i}^{2}+3 g \alpha_{n} T_{n}\right)\right] \cos \alpha_{n} x } \\
& +\mathrm{i} \sum_{n=1}^{\infty} \frac{\widehat{\theta} p_{n}}{\left(W_{1}-g \alpha_{n} T_{n}\right)^{2}+W_{2}^{2}}\left[\Omega_{i}\left(\left(W_{1}-g \alpha_{n} T_{n}\right)\left(g-\frac{2 g}{C_{n}}-W_{1} H_{0}\right)-W_{2}^{2} H_{0}\right)\right. \\
& \left.-g W_{2} \Omega_{r}\left(1-\frac{2}{C_{n}}-T_{n} \alpha_{n} H_{0}\right)\right] \cos \alpha_{n} x
\end{aligned}
$$

where

$$
W_{1}=\Omega_{r}^{2}-\Omega_{i}^{2}, \quad \text { and } \quad W_{2}=2 \Omega_{r} \Omega_{i},
$$

and we have introduced $h_{0}+d_{2}=h_{0}-l=H_{0}$ for brevity.

\subsection{The characteristic function}

The characteristic function for this problem is found by substituting $\widehat{\phi}$ into the linearised vessel equation (3.9). This requires the evaluation of the two integrals

$$
\begin{aligned}
\left.\int_{0}^{L}\left(x+d_{1}\right) \widehat{\phi}_{y}\right|_{y=h_{0}} d x & =-2 \sum_{n=0}^{\infty} \frac{1}{\alpha_{n}}\left(a_{n} S_{n}-\frac{2 \widehat{\theta} p_{n} \omega}{\alpha_{n}} C_{n}\right) \\
& =2 \widehat{\theta} \omega \sum_{n=0}^{\infty} \frac{p_{n}}{\alpha_{n}\left(\omega^{2}-g \alpha_{n} T_{n}\right)}\left(\frac{2 \omega^{2}}{\alpha_{n} C_{n}}-T_{n}\left(g-\omega^{2} H_{0}\right)\right)
\end{aligned}
$$

where $S_{n}=\sinh \alpha_{n} h_{0}$, and

$$
\begin{aligned}
\int_{0}^{L} \int_{0}^{h_{0}}\left[\left(y+d_{2}\right) \widehat{\phi}_{x}-\left(x+d_{1}\right) \widehat{\phi}_{y}\right] d y d x= & \sum_{n=0}^{\infty} \frac{2 \omega \widehat{\theta} p_{n}}{\alpha_{n}^{2}\left(\omega^{2}-g \alpha_{n} T_{n}\right)}\left[T_{n} \alpha_{n} H_{0}\left(\omega^{2} H_{0}-g\right)\right. \\
& -2 \omega^{2}\left(H_{0}+\frac{2 T_{n}}{\alpha_{n}}\right)-2 d_{2}\left(\omega_{2}-g \alpha_{n} T_{n}\right) \\
& \left.+\frac{2}{C_{n}}\left(3 g\left(C_{n}-1\right)+2 \omega^{2} H_{0}\right)\right]
\end{aligned}
$$

where $d_{1}=-L / 2$ has been used to simplify the expressions.

Therefore the characteristic function can be written as

$$
\Delta(\omega)=P(\omega) D(\omega)=0
$$


where

$$
\begin{aligned}
D(\omega)= & {\left[-\omega^{2}\left(m_{v}+m_{f}-\frac{m_{f} L^{2}}{12 l^{2}}-\frac{m_{f} h_{0}}{l}+\frac{m_{f} h_{0}^{2}}{3 l^{2}}\right)+\frac{g}{l}\left(m_{v}+m_{f}-\frac{m_{f} h_{0}}{2 l}\right)-\frac{g L^{2} m_{f}}{6 l^{2} h_{0}}\right.} \\
& +\frac{8 \omega^{2} m_{f}}{h_{0} L^{2} l^{2}} \sum_{n=0}^{\infty} \frac{1}{\alpha_{n}^{3}\left(\omega^{2}-g \alpha_{n} T_{n}\right)}\left[\frac{T_{n}}{\omega^{2}}\left(\omega^{4}\left(h_{0}-l\right)^{2}-g^{2}\right)-\frac{2 \omega^{2}}{\alpha_{n}}\left(h_{0}-l+\frac{2 T_{n}}{\alpha_{n}}\right)\right. \\
& \left.\left.+\frac{2 l}{\alpha_{n}}\left(\omega^{2}-g \alpha_{n} T_{n}\right)+\frac{2}{C_{n} \alpha_{n}}\left(g\left(3 C_{n}-2\right)+2 \omega^{2}\left(h_{0}-l\right)\right)\right]\right] \prod_{n=0}^{\infty}\left(\omega^{2}-g \alpha_{n} T_{n}\right), \\
P(\omega)= & \prod_{n=1}^{\infty}\left(\omega^{2}-g \beta_{n} \widehat{T}_{n}\right),
\end{aligned}
$$

and we have substituted $d_{2}=-l, p_{n}=-4 /\left(L \alpha_{n}^{2}\right)$ and used the fact that $m_{f}=\rho h_{0} L$. Unlike for the Cooker experiment, here $\omega^{2}-g \alpha_{n} T_{n}=0$ can lead to a root of the characteristic function, so we cannot neglect these terms in $D(\omega)$. An alternative derivation of the characteristic function can be found in Moiseyev and Rumyantsev (1968) (see also Chapter 5 of Faltinsen and Timokha (2009) for the approach based on added mass coefficients).

We can express the characteristic function in terms of the non-dimensional variables proposed by Cooker (1994) which are $R$, defined in (1.2), and

$$
G=\frac{L^{2}\left(m_{v}+m_{f}\right)}{4 h_{0} m_{f} l}=\frac{L\left(m_{v}+m_{f}\right)}{4 \delta m_{f} l},
$$

with additional parameters,

$$
\begin{array}{r}
\delta=\frac{h_{0}}{L}, \quad \frac{l}{L}=\frac{(1+R)}{4 \delta G}, \quad s=\frac{\omega L}{2 \sqrt{g h_{0}}}, \\
\gamma_{n}=\alpha_{n} L=(2 n+1) \pi, \quad \widehat{\gamma}_{n}=\beta_{n} L=2 n \pi, \\
\sigma_{n}=\frac{L}{g}\left(\omega^{2}-g \alpha_{n} T_{n}\right)=4 \delta s^{2}-\gamma_{n} T_{n}, \quad \widehat{\sigma}_{n}=4 \delta s^{2}-\widehat{\gamma}_{n} \widehat{T}_{n}, \\
T_{n}=\tanh \left(\gamma_{n} \delta\right), \quad \widehat{T}_{n}=\tanh \left(\widehat{\gamma}_{n} \delta\right), \quad C_{n}=\cosh \left(\gamma_{n} \delta\right) .
\end{array}
$$

This leads to the non-dimensional characteristic function for the dimensionless frequency $s$

where

$$
\Delta(s)=P(s) D(s)
$$

$$
\begin{aligned}
D(s)= & {\left[G-\frac{2 \delta^{2} G^{2}}{(1+R)^{2}}-\frac{2 G^{2}}{3(1+R)^{2}}-s^{2}\left(1+R-\frac{4 \delta^{2} G^{2}}{3(1+R)^{2}}-\frac{4 \delta^{2} G}{1+R}+\frac{16 \delta^{4} G^{2}}{3(1+R)^{2}}\right)\right.} \\
& +32 \sum_{n=0}^{\infty} \frac{1}{\gamma_{n}^{3} \sigma_{n}}\left[T_{n}\left(s^{4}\left(\frac{4 \delta^{2} G}{1+R}-1\right)^{2}-\frac{G^{2}}{(1+R)^{2}}\right)-\frac{8 \delta s^{4} G}{\gamma_{n}(1+R)}\left(\frac{4 \delta^{2} G}{1+R}-1+\frac{8 \delta T_{n} G}{\gamma_{n}(1+R)}\right)\right. \\
& \left.\left.+\frac{2 s^{2} \sigma_{n} G}{\gamma_{n}(1+R)}+\frac{8 \delta s^{2} G}{C_{n} \gamma_{n}(1+R)}\left(\frac{G}{1+R}\left(3 C_{n}-2\right)+2 s^{2}\left(\frac{4 \delta^{2} G}{1+R}-1\right)\right)\right]\right] \prod_{n=0}^{\infty} \sigma_{n}, \\
P(s)= & \prod_{n=1}^{\infty} \widehat{\sigma}_{n} .
\end{aligned}
$$

The characteristic function is solved numerically in $\S 3.3$ for those complex roots $s$ which have positive real part, for a given parameter set $(G, R, \delta)$. 


\subsection{The Shallow Water Limit}

The shallow water limit form of the characteristic function $\Delta^{\mathrm{SW}}(s)$ can be calculated by letting $\delta \rightarrow 0$ in (3.17) and noting that

$$
\begin{array}{ll}
\sigma_{n} \rightarrow \delta \Gamma_{n}=\delta\left(4 s^{2}-\gamma_{n}^{2}\right), & T_{n} \rightarrow \delta \gamma_{n}, \\
\widehat{\sigma}_{n} \rightarrow \delta \widehat{\Gamma}_{n}=\delta\left(4 s^{2}-\widehat{\gamma}_{n}^{2}\right), & \widehat{T}_{n} \rightarrow \delta \widehat{\gamma}_{n},
\end{array}
$$

where the above equations define $\Gamma_{n}$ and $\widehat{\Gamma}_{n}$, to give

$$
\begin{aligned}
D^{\mathrm{SW}}(s)= & {\left[G-s^{2}(1+R)-\frac{2 G^{2}}{3(1+R)^{2}}+8\left(s^{4}-\frac{G^{2}}{(1+R)^{2}}\right) \sum_{n=0}^{\infty} \frac{4}{\gamma_{n}^{2} \Gamma_{n}}\right.} \\
& \left.+\frac{16 s^{2} G}{1+R}\left(\frac{G}{1+R}-s^{2}\right) \sum_{n=0}^{\infty} \frac{16}{\gamma_{n}^{4} \Gamma_{n}}+\frac{64 s^{2} G}{1+R} \sum_{n=0}^{\infty} \frac{1}{\gamma_{n}^{4}}\right] \cos (s) .
\end{aligned}
$$

We can replace $\prod_{n=0}^{\infty} \Gamma_{n}$ with $\cos (s)$ because as $\delta \rightarrow 0$ the roots of $\prod_{n=0}^{\infty} \Gamma_{n}$ occur at $s=\frac{1}{2} \gamma_{n}$ which correspond to the roots of $\cos (s)$. Similarly in $P(s)$ we can replace $\prod_{n=0}^{\infty} \widehat{\Gamma}_{n}$ with $\sin (s)$.

Now by noting that

$$
\frac{4}{\gamma_{n}^{2} \Gamma_{n}}=\frac{1}{s^{2} \gamma_{n}^{2}}+\frac{1}{s^{2} \Gamma_{n}}, \quad \text { and } \quad \frac{16}{\gamma_{n}^{4} \Gamma_{n}}=\frac{4}{s^{2} \gamma_{n}^{4}}+\frac{1}{s^{4} \gamma_{n}^{2}}+\frac{1}{s^{4} \Gamma_{n}},
$$

as well as

$$
\sum_{n=0}^{\infty} \frac{1}{\gamma_{n}^{2}}=\frac{1}{8}, \quad \sum_{n=0}^{\infty} \frac{1}{\gamma_{n}^{4}}=\frac{1}{96}, \text { and } \sum_{n=0}^{\infty} \frac{1}{\Gamma_{n}}=-\frac{\tan (s)}{8 s},
$$

then the characteristic function can be written as

$$
\Delta^{\mathrm{SW}}(s)=\sin (s) \cos (s)\left[G-s^{2} R-s \tan (s)+\frac{G}{1+R}\left(\frac{G}{1+R}-2 s^{2}\right)\left(\frac{1}{s^{2}}-\frac{\tan (s)}{s^{3}}\right)\right]=0 .
$$

This characteristic function can be compared to the shallow water characteristic function for the Cooker experiment given by

$$
\sin (s) \cos (s)\left[G-s^{2} R-s \tan (s)\right]=0 .
$$

Note that in the Cooker experiment it is impossible for an unstable transition of the form in figure 2 to exist.

\subsection{Numerical Solutions of the Characteristic Function}

In this section we will show that the characteristic function (3.17) exhibits both real (stable) and complex (unstable) roots for the non-dimensional frequency $s$. From (3.17) it is clear that the symmetric modes, which are solutions to $P(s)=0$, have solutions $s=\sqrt{\widehat{\gamma}_{n} \widehat{T}_{n} /(4 \delta)}$, but due to the implicit form of $D(s)$ we cannot make any analytical progress in finding the roots of the anti-symmetric modes and instead we search for these roots numerically. The real roots of $D(s)=0$ are found by plotting $D(s)$ for a fixed 


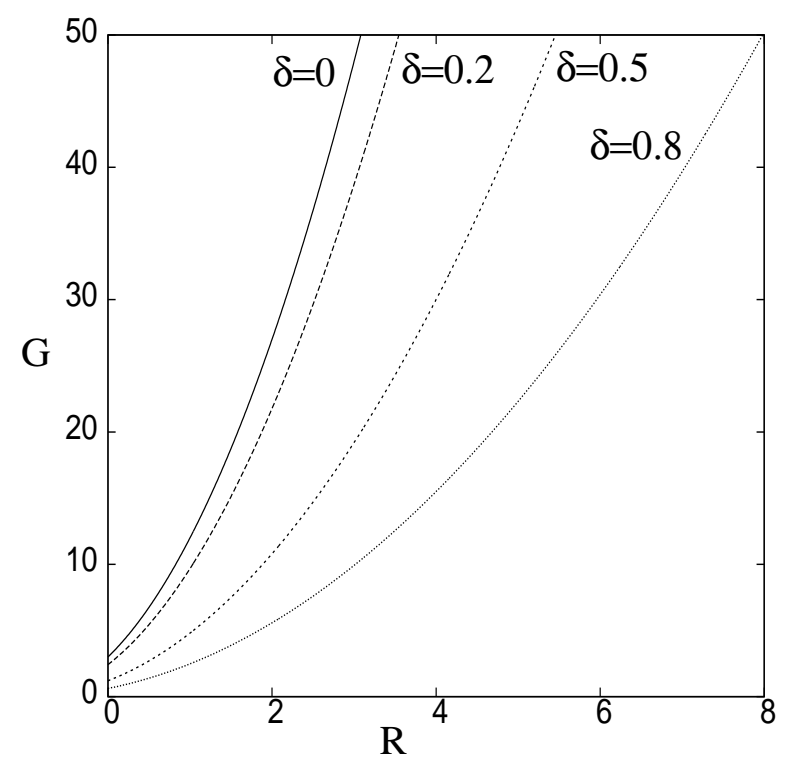

Figure 3: Plot of the neutral stability contour (3.21) in the $(R, G)$ parameter space for $\delta=0.8,0.5,0.2$ and 0 . Above the contour the solutions are unstable, while below the contour the solutions are stable.

set of parameters $(G, R, \delta)$ and calculating the values where this function crosses the real axis.

To find the complex roots, we note that $D(s)$ in (3.18) is an even function of $s$, hence we solve (3.18) for $s^{2}$, and when this quantity is negative we get purely imaginary roots. The unstable solutions are restricted to a particular region of the 3D parameter space, which can be determined by solving (3.18) at $s^{2}=0$, which is the point at which instability occurs in the characteristic function. The result of this calculation gives a neutral stability surface in parameter space which separates the stable and unstable solutions. By setting $s=0$ in (3.18) it can be shown that the neutral stability contour is analytically given by

$$
G=\frac{3(1+R)^{2}}{1+6 \delta^{2}}
$$

This neutral stability contour is shown in the $(R, G)$ - plane in figure 3 for $\delta=0.8,0.5,0.2$ and 0 . Above this contour the solutions are unstable, while below the contour the solutions are stable. Note, we only consider results for $G \geq 0$, which corresponds to $l \geq 0$, which means that the pivot point never lies below the bottom of the vessel. Thus we have the restriction that $\widehat{l} \geq-d$.

In terms of dimensional variables this says there is an instability in the system if (1.1) is satisfied. The consequence of (1.1) is that, there is an instability in the system as the length of the rod about which the tank is pivoting tends to zero. We can also deduce from (1.1) that for fluids of depth $\delta>1 / \sqrt{6(1+2 R)}$ the pivot point would lie inside the fluid domain at the instability point, i.e. the pivot point lies inside the fluid before the instability is reached. However, for $\delta<1 / \sqrt{6(1+2 R)}$ the instability occurs before the pivot point meets the top of the fluid layer.

Suppose $s=S_{r}+\mathrm{i} S_{i}$. Then the values of $S_{r}(G)$ and $-S_{i}(G)$ for fixed values of $R$ and $\delta$ are plotted in figure 4 . This figure shows that the non-dimensional growth rate increases 
(a)
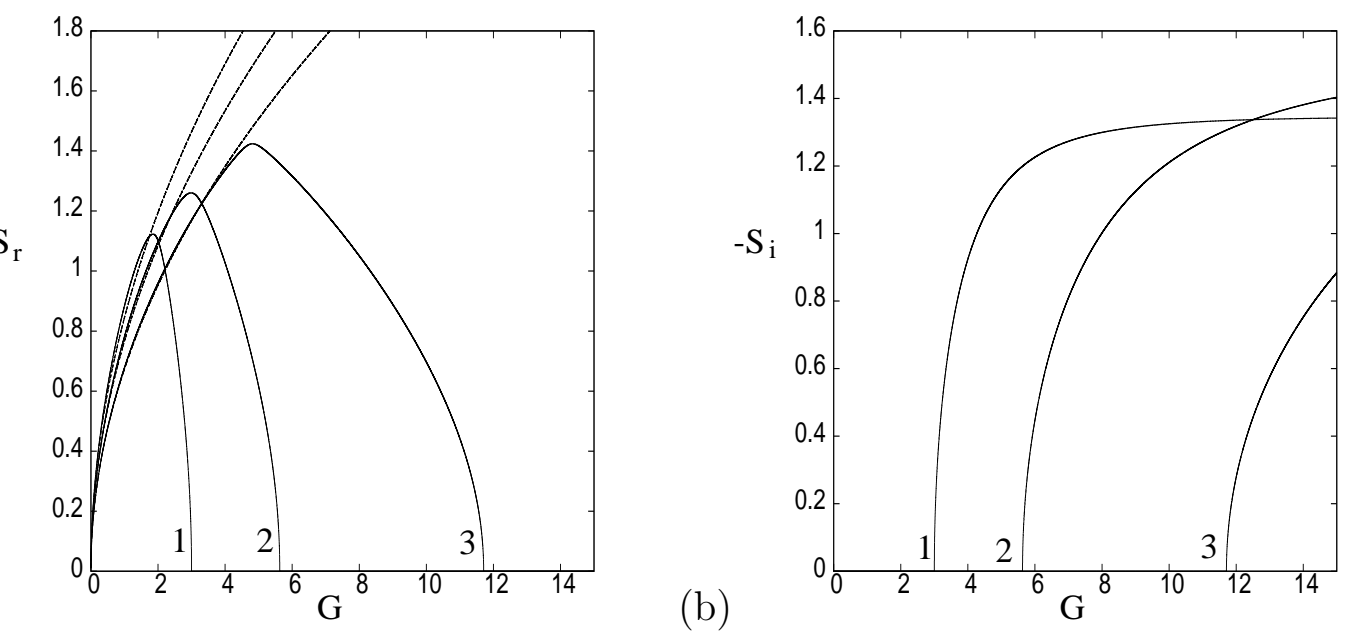

Figure 4: (a) $S_{r}(G)$ and (b) $-S_{i}(G)$ for the fundamental mode plotted for $(R, \delta)=$ $(0.4,0.4),(0.7,0.3)$ and $(1.2,0.2)$ numbered $1-3$ respectively. the dashed lines in panel (a) correspond to the pendulum frequency in (3.22).

rapidly once the instability contour is reached, and figure 4(a) shows that as $G$ is increased from 0 , the frequency increases and reaches a maximum value before decreasing towards the instability contour. This can more clearly be seen in the contour plots in figure 5 . In figure 4(a) the dashed lines correspond to the non-dimensional frequencies obtained by modelling the dry vessel as a point mass. In this case $\omega=\sqrt{l / g}$, therefore

$$
s=\sqrt{\frac{G}{1+R}}
$$

Hence, the results in figure 4(a) show that for small values of $G$ the vessel frequency is independent of the fluid motion and oscillates as a dry vessel. However, beyond some critical value of $G$ where $S_{r}$ obtains its maximum value, the fluid motion becomes significant in determining the coupled vessel frequency and the coupled frequency decreases.

The contour plots in figure 5 show that the largest values of $S_{r}$ occur for small values of $\epsilon$, and that the range of $G$ values over which $S_{r}>0.5$, say, reduces with increasing $\epsilon$. However, one should note that the dimensional frequency is given by $\omega=2 \sqrt{g h_{0}} / L$ and hence is proportional to $\delta^{1 / 2}$. Therefore, although $S_{r}$ increases as $\delta$ decreases, this may not be the case for the dimensional frequency $\omega$.

Another interesting feature of this experimental system occurs close to the frequencies of the free anti-symmetric sloshing modes, which occur with non-dimensional frequencies

$$
s_{n}^{(a)}=\frac{1}{2}(2 n+1) \pi \sqrt{\frac{\tanh ((2 n+1) \pi \delta)}{(2 n+1) \pi \delta}}, \quad n=0,1,2, \ldots .
$$

These correspond to values of $s$ where $\sigma_{n}=0$ in (3.18). At $s=s_{n}^{(a)}, D\left(s_{n}^{(a)}\right)=0$ if

$$
G=\frac{(1+R) \gamma_{n}}{4 \delta} \frac{S_{n}\left(\delta S_{n} \gamma_{n}-C_{2}+2\right)}{\delta^{2} S_{n}^{2} \gamma_{n}^{2}-2 \delta S_{n} C_{n} \gamma_{n}+4 \delta S_{n} \gamma_{n}+\left(C_{n}-2\right)^{2}}
$$

where $S_{n}=\sinh \left(\gamma_{n} \delta\right)$. 

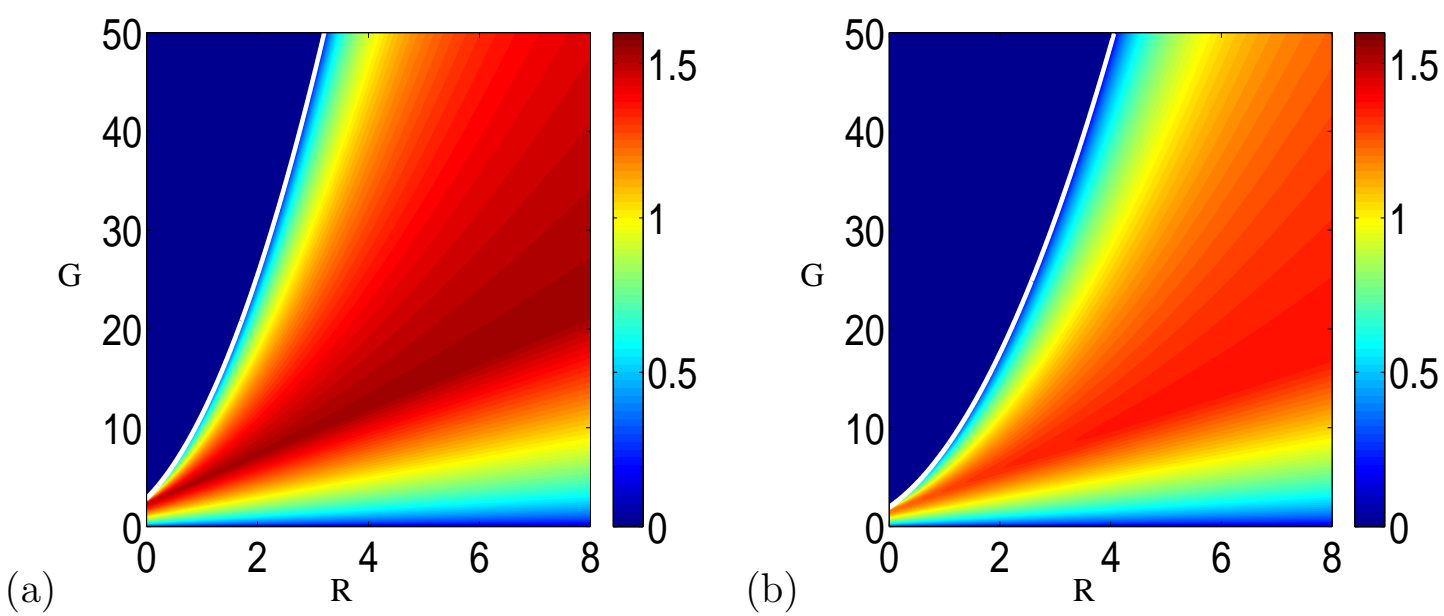

(b)

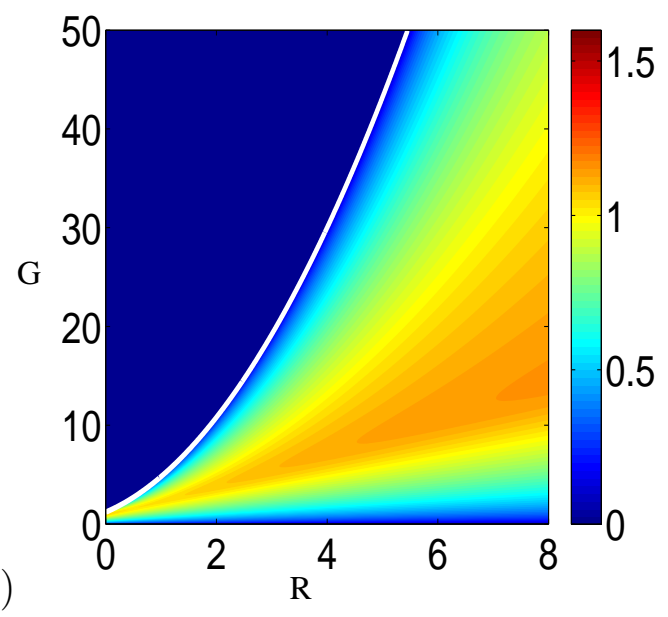

Figure 5: (colour online) Contour plots of $S_{r}(R, G)$ for the fundamental mode for (a) $\delta=0.1$, (b) $\delta=0.3$ and (c) $\delta=0.5$. In each panel the white line indicates the neutral stability contour.

This particular value of $G$ does not lead to a double root of the characteristic function, as one of the roots is removable, but close to this value there exists two real roots of $D(s)=0$ with similar frequencies, as shown in figure 6 for the case when $n=0$. The result with $G=6.4889$ corresponds to the critical value of $G$ in (3.23), and as can be seen, there is only the one root of the characteristic function, but either side of this value there exists two real roots.

\section{$3.4 \quad 1: 1$ internal resonance}

Earlier in this section we showed that the natural frequency of the symmetric modes is determined independently of the anti-symmetric modes, and hence the vessel motion in the characteristic function. Therefore, this system contains an internal $1: 1$ resonance where both independent modes have the same frequency. This occurs when the two factors of the characteristic function (3.17) vanish simultaneously

$$
P(s)=0 \quad \text { and } \quad D(s)=0,
$$




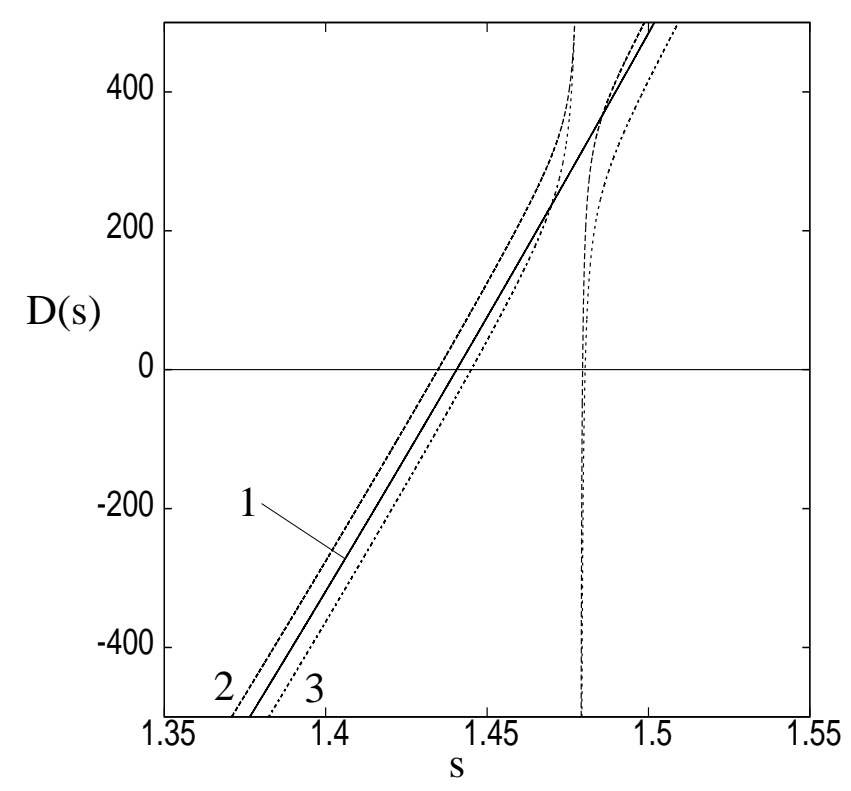

Figure 6: Plot of $D(s)$ for $\delta=0.2, R=2$ and $G=6.4889,6.4$ and 6.6 numbered 1-3 respectively. The value $G=6.4889$ is the critical value of $G$ giving a single root when $\sigma_{0}=0$.

and the symmetric sloshing modes have the same frequency as one of the coupled vessel sloshing modes. The zeros of the symmetric modes occur when $\widehat{\sigma}_{n}=0$, i.e. at

$$
s=s_{j}^{(s)}=j \pi \sqrt{\frac{\tanh (2 j \pi \delta)}{2 j \pi \delta}}, \quad j=1,2,3, \ldots .
$$

At this value of $s$, for a $1: 1$ resonance to exist the second term in the characteristic function must satisfy $D\left(s_{j}^{(s)}\right)=0$, which from (3.18) leads to a quadratic equation for $G(R, \delta)$. The curve in the $(R, G)$-plane along which the $1: 1$ resonance occurs is shown in figure 7 for various values of $\delta$ and for (a) $j=1$ and (b) $j=2$. Note that in order to determine whether or not a $1: 1$ resonance is possible, the results in figure 7 need to be taken in conjunction with those in figure 3 to see whether the $1: 1$ resonance curve lies in the stable part of parameter space. In the shallow water limit, $\delta \rightarrow 0$, the position of the $1: 1$ resonance occurs at $s_{j}^{(s)}=j \pi$, and from (3.20) the corresponding quadratic equation for $G$ becomes

$$
G^{2}+G\left(s_{j}^{(s)}\right)^{2}\left(R^{2}-1\right)-\left(s_{j}^{(s)}\right)^{4} R(1+R)^{2}=0
$$

which has solutions

$$
G=\left(s_{j}^{(s)}\right)^{2}(1+R) \quad \text { and } \quad G=-\left(s_{j}^{(s)}\right)^{2} R(1+R),
$$

the second of which can be ignored as we have $G>0$. This corresponds to a straight line in the $(R, G)$-plane, and is similar to the Cooker problem 1:1 resonance where $G=\left(s_{j}^{(s)}\right)^{2} R$ (Alemi Ardakani et al., 2012a). As $\delta$ is increased, then at some critical 

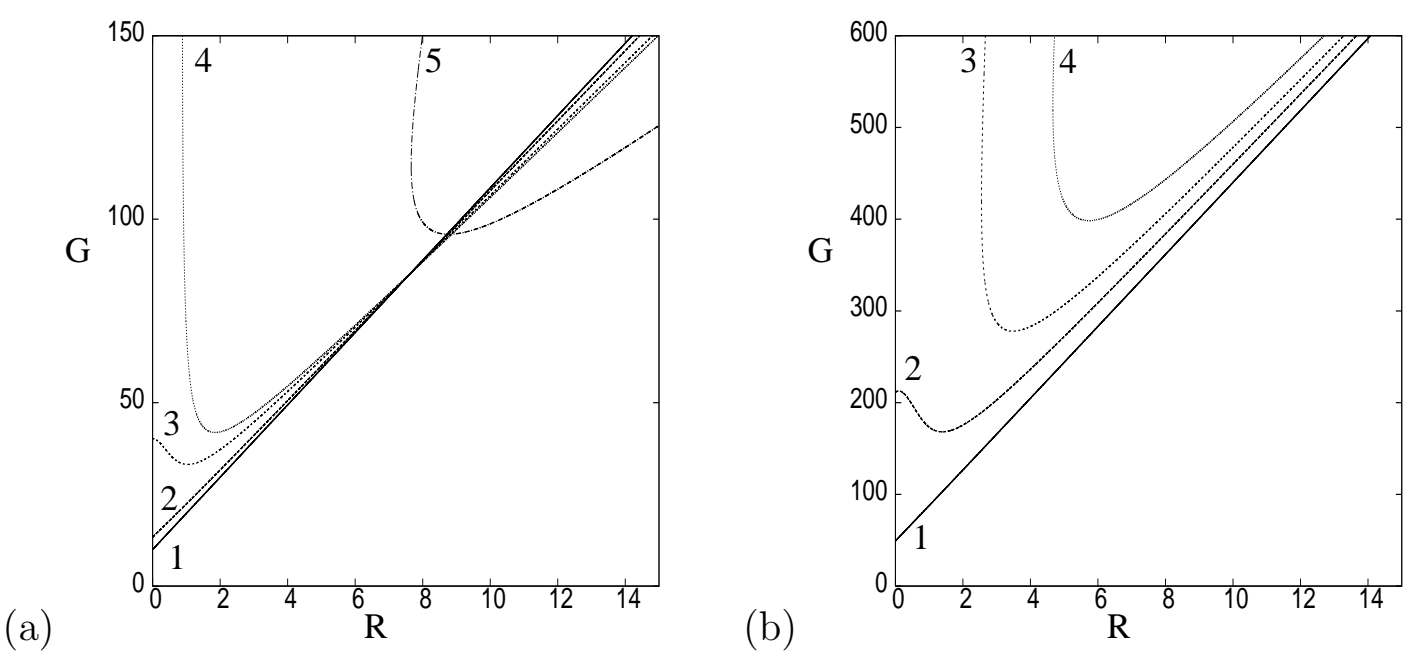

Figure 7: Plot of (a) the first $(j=1)$ and (b) the second $(j=2)$ resonance curves in the $(R, G)$ - plane. In panel (a) the curves 1-5 correspond to $\delta=0.01,0.05,0.08,0.09$ and 0.2 respectively, while in panel (b) the curves $1-4$ correspond to $\delta=0.01,0.02,0.025$ and 0.03 respectively.

depth $\delta_{1}$ there becomes two positive roots for $G$ giving a curve, such as curve 4 in figure 7 (a), which is closed near $R=0$. This contour then moves off to large values of $R$ as $\delta$ is increased further. For $j=1$ and 2 , this critical depth is $\delta_{1}=0.0896$ and $\delta_{1}=0.0221$ respectively.

When these results are compared with those for the Cooker experiment (figure 4 of Alemi Ardakani et al. (2012a)) we see that here the $1: 1$ resonance positions for the pendulum problem occur at much larger values of $G$ for the same mass ratio $R$. Therefore, the 1:1 resonance is unlikely to be observed for the pendulum problem for an experimentally realistic setup. However, if such an experimental setup was possible a weakly nonlinear analysis of the $1: 1$ resonance values would determine if any have heteroclinic orbits connecting the symmetric and anti-symmetric modes leading to an energy transfer mechanism.

\section{Homoclinic Orbit}

The governing equations are Hamiltonian, and the Hamiltonian formulation is obtained by Legendre transform of the Lagrangian in $\S 2$. The specifics of the Hamiltonian structure are not needed, but they assure that the bifurcations will follow the Hamiltonian structure. A Hamiltonian system with a saddle-centre transition of eigenvalues as in Figure 2 always leads to homoclinic bifurcation (Meyer and Hall, 1992; Arnold et al., 1993; Bridges, 2012). To see this first consider the case of a planar parameter-dependent Hamiltonian system as in this case the theory is exact,

$$
-\dot{p}=\frac{\partial H}{\partial q} \quad \text { and } \quad \dot{q}=\frac{\partial H}{\partial p},
$$

where $H(q, p, \alpha)$ is a parameter-dependent Hamiltonian function. Now, suppose that the linearisation about the trivial solution has a saddle-centre transition of eigenvalues: for 
some value of $\alpha$, denoted by $\alpha_{0}$, the Hessian is degenerate: $\operatorname{det}\left(\left.D^{2} H\right|_{\alpha=\alpha_{0}}\right)=0$, where

$$
D^{2} H:=\left[\begin{array}{ll}
\frac{\partial^{2} H}{\partial q^{2}} & \frac{\partial^{2} H}{\partial q \partial p} \\
\frac{\partial^{2} H}{\partial p \partial q} & \frac{\partial^{2} H}{\partial p^{2}}
\end{array}\right] .
$$

In the weakly nonlinear problem near the transition, the generation of the homoclinic orbit is shown by transforming the nonlinear system (4.1) into nonlinear normal form (Meyer and Hall, 1992; Arnold et al., 1993; Bridges, 2012). Introduce new coordinates $(\widehat{q}, \widehat{p})$. Then nonlinear transformations lead to

$$
-\widehat{p}_{t}=\mu-\frac{1}{2} \kappa \widehat{q}^{2}+\cdots \quad \text { and } \quad \widehat{q}_{t}=\widehat{p}+\cdots,
$$

where $\mu=\left(\alpha-\alpha_{0}\right)$ is an unfolding parameter, and $\kappa$ is a parameter depending on the nonlinearity of the original system (various formulae for $\kappa$ are given in Bridges (2012)), and the dots are terms of higher order in $(\widehat{q}, \widehat{p})$. The phase portrait for (4.3) is shown

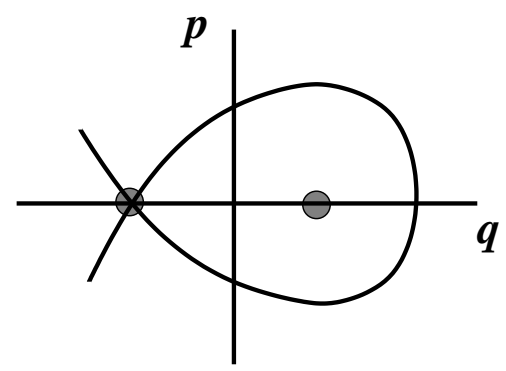

Figure 8: The homoclinic orbit appearing in the nonlinear unfolding of the saddle-center transition. The two dots represent equilibrium points for the system.

in figure 8. It is a homoclinic orbit formed around a pair of equilibria, one of which is hyperbolic (unstable), the other elliptic (stable), and these two points coalesce as $\alpha \rightarrow \alpha_{0}$. In the case of a planar Hamiltonian system, this theory is exact and the homoclinic orbit is persistent.

In the present case the system is infinite dimensional and so transformations will still lead to (4.3) on a two-dimensional subspace, but there will be an infinite-dimensional complementary space. The system will be of the form

$$
\begin{aligned}
-\widehat{p}_{t} & =\mu-\frac{1}{2} \kappa \widehat{p}^{2}+\cdots, \\
\widehat{q}_{t} & =\widehat{p}+\cdots, \\
Z_{t} & =f(Z, \widehat{q}, \widehat{p}) .
\end{aligned}
$$

However, due to the large dimension of the phase space, the stable and unstable manifolds can not intersect transversely, and so the homoclinic orbit will not be a persistent or true homoclinic orbit. On the other hand, it will still produce complex dynamics due to the breakup of the homoclinic orbit by the higher order terms. This complex dynamics is interesting, and the implications will be explored in follow-up work on the nonlinear problem. In terms of a mechanical system such as that of the TLD damping torsional bridge oscillations, this complex dynamics would lead to unpredictable oscillations of the fluid in the TLD. This fluid motion would then feedback onto the structure potentially leading to difficult driving conditions for vehicles crossing the bridge. 


\section{Conclusions and Discussion}

This paper demonstrated that the coupled pendulum sloshing problem, consisting of a vessel of inviscid fluid suspended by a single rigid rod which is allowed to rotate in the $(x, y)$-plane, exhibits unstable solutions if the length of the rod is shorter than some critical length. In terms of dimensional variables instability occurs in the system if

$$
(1+R) l<\frac{1}{2} h_{0}+\frac{1}{12} \frac{L^{2}}{h_{0}},
$$

and moreover, if $\delta=h_{0} / L<1 / \sqrt{6(1+2 R)}$ then the instability occurs in the system with the pivot point of the vessel above the surface of the fluid. The instability transition is associated with the emergence of homoclinic behaviour in the weakly nonlinear problem. The existence of this instability would have a detrimental effect on mechanical systems which use a rotational tuned liquid damper to stabilise the structure, such as on bridges destabilized by aerodynamic effects (Xue et al., 2000; Chen et al., 2008).

For the stable sloshing modes, we were able to show that a $1: 1$ fluid resonance exists in the system, where the symmetric sloshing modes oscillate with the same frequency as the coupled vessel and anti-symmetric sloshing modes, as was the case in the Cooker bi-linear pendulum experiment (Alemi Ardakani et al., 2012a). In the Cooker experiment, these $1: 1$ resonances could be found along straight lines in the $(R, G)$-plane. However, in the pendulum experiment we found that for values of $\delta$ below some critical value $\delta_{1}$, the $1: 1$ resonance could be found along a single curved contour in the $(R, G)$-plane, but above $\delta_{1}$ this contour became closed close to $R=0$ and had two branches which extended to infinity. The significance of this is that the parameter space for the pendulum problem contains more values at which the $1: 1$ resonance exists, but these values lie in regions of parameter space which would be difficult to achieve in experimental setups. However, if such a setup were achieved, then constructing a weakly nonlinear analysis about these $1: 1$ resonances would show whether or not a heteroclinic orbit exists between the purely symmetric and the purely anti-symmetric modes, leading to an energy transfer between these modes (Turner and Bridges, 2013).

\section{Acknowledgments}

This work is supported by the EPSRC under grant number EP/K008188/1.

\section{Appendix -}

\section{A Comparison of the Lagrangian (2.7) with Moiseyev and Rumyantsev (1968)}

Equation (5-65) in Moiseyev and Rumyantsev (1968) gives the kinetic energy for the system as

$$
T=\frac{J \dot{\theta}}{2}+\dot{\theta} \rho \int_{V} \nabla \phi^{M} \cdot \nabla \phi^{*} d V+\frac{\rho}{2} \int_{V}\left(\nabla \phi^{M}\right)^{2} d V
$$


and the equation after (5-65) gives the potential energy as

$$
\Pi=\frac{l_{0} M_{0} g \theta^{2}}{2}+\rho g \int_{V} Z d V .
$$

Here $M_{0}$ is the mass of the vessel, $l_{0}$ is the distance between the vessel centre of mass to the pivot point, $\phi^{M}$ is the velocity potential, $\phi^{*}$ is the so called Stokes-Zhukovskiy potential, $V$ is the fluid volume inside the vessel, $g$ is gravity and $J=J_{0}+m$ is the moment of inertia of the vessel plus

$$
m=\rho \int_{V}\left(\nabla \phi^{*}\right)^{2} d V
$$

which is the added moment of inertia by the fluid. The function $Z=(z-l) \cos \theta+y \sin \theta$ where $l$ is the distance from the pivot to the average position of the free-surface, $z$ is a coordinate pointing vertically upwards and $y$ is a horizontal coordinate.

Converting notation from Moiseyev and Rumyantsev (1968) to our notation and evaluating for a rectangular vessel with a centre of mass at the centre of the base of the vessel, we have the following changes of notation

$$
\begin{aligned}
M_{0} & \rightarrow m_{v}, \quad l_{0} \rightarrow-d_{2}, \quad z \rightarrow y-h_{0}, \\
y & \rightarrow x+d_{1}, \quad l \rightarrow-\left(d_{2}+h_{0}\right), \quad \phi^{*}=-\left(x+d_{1}\right)\left(y+d_{2}\right), \\
J_{0} & =m_{v} d_{2}^{2}, \quad m=\rho \int_{0}^{L} \int_{0}^{h}\left[\left(x+d_{1}\right)^{2}+\left(y+d_{2}\right)^{2}\right] d y d x, \\
\frac{\partial \phi^{M}}{\partial x} & \rightarrow u, \quad \frac{\partial \phi^{M}}{\partial y} \rightarrow-v .
\end{aligned}
$$

Therefore substituting these into the kinetic and potential energies gives

$$
\begin{aligned}
T= & \frac{\dot{\theta}^{2}}{2}\left[m_{v} d_{2}^{2}+\rho \int_{0}^{L} \int_{0}^{h}\left[\left(x+d_{1}\right)^{2}+\left(y+d_{2}\right)^{2}\right] d y d x\right] \\
& +\rho \int_{0}^{L} \int_{0}^{h}\left[\frac{1}{2}\left(u^{2}+v^{2}\right)-\dot{\theta}\left(y+d_{2}\right) u+\dot{\theta}\left(x+d_{1}\right) v\right] d y d x
\end{aligned}
$$

and

$$
\Pi=-\frac{d_{2} m_{v} g \theta^{2}}{2}+\rho g \int_{0}^{L} \int_{0}^{h}\left[\left(y+d_{2}\right) \cos \theta+\left(x+d_{1}\right) \sin \theta\right] d y d x .
$$

The first term of $\Pi$ is the small $\theta$ expansion of $d_{2} m_{v} g \cos \theta$ minus the irrelevant constant term and the higher order terms, so we can write

$$
\Pi=d_{2} m_{v} g \cos \theta+\rho g \int_{0}^{L} \int_{0}^{h}\left[\left(y+d_{2}\right) \cos \theta+\left(x+d_{1}\right) \sin \theta\right] d y d x .
$$

Thus the Lagrangian of Moiseyev and Rumyantsev (1968) is

$$
\mathscr{L}^{M}=T-\Pi=\mathscr{L},
$$

which corresponds to (2.7) and so the vessel equations are equivalent.

It is also possible to show using the above transformation that the linear equations given in (5-68) of Moiseyev and Rumyantsev (1968) transform into the linear equations given in $\S 3$. 


\section{References}

Abramson, H. N., Chu, W. H., and Ransleben, JR., G. E. (1961). Representation of fuel sloshing in cylindrical tanks by an equivalent mechanical model. Am. Rocket Soc., 31, $1697-1705$.

Adee, B. H. and Caglayan, I. (1982). The effects of free water on deck on the motions and stability of vessels. In Proc. Second Inter. Conf. Stab. Ships and Ocean Vehicles, Tokyo. SNAME, Springer.

Alemi Ardakani, H. (2010). Rigid-body motion with interior shallow-water sloshing. PhD Thesis, University of Surrey, Guildford, UK.

Alemi Ardakani, H. and Bridges, T. J. (2010). Dynamic coupling between shallow-water sloshing and horizontal vehicle motion. Europ. J. Appl. Math, 21, 479-517.

Alemi Ardakani, H. and Bridges, T. J. (2011). Shallow-water sloshing in vessels undergoing prescribed rigid-body motion in three dimensions. J. Fluid Mech., 667, 474-519.

Alemi Ardakani, H., Bridges, T. J., and Turner, M. R. (2012a). Resonance in a model for Cooker's sloshing experiment. Euro. J. Mech. B/Fluids, 36, 25-38.

Alemi Ardakani, H., Bridges, T. J., and Turner, M. R. (2012b). Resonance in a model for Cooker's sloshing experiment - extended version. Technical Report, University of Surrey Repository. http://epubs.surrey.ac.uk/713639/.

Arnold, V. I., Kozlov, V. V., and Neishtadt, A. I. (1993). Mathematical Aspects of Classical and Celestial Mechanics. Springer-Verlag (Berlin).

Bridges, T. J. (2012). Geometric lift of paths of Hamiltonian equilibria and homoclinic bifurcation. Int. J. Bifurcation Chaos, 22, 1250304.

Caglayan, I. and Storch, R. L. (1982). Stability of fishing vessels with water on deck: a review. J. Ship Research, 26, 106-116.

Chen, S. and Cai, C. (2004). Accident assessment of vehicles on long-span bridges in windy environments. Journal of Wind Engineering and Industrial Aerodynamics, 92(12), 9911024.

Chen, S., Chang, C., and Cai, C. (2008). Study on Stability Improvement of Suspension Bridge with High-Sided Vehicles under Wind using Tuned-Liquid-Damper. Journal of Vibration and Control, 14(5), 711-730.

Cooker, M. J. (1994). Water waves in a suspended container. Wave Motion, 20, 385-395.

Dillingham, J. (1981). Motion studies of a vessel with water on deck. Wave Motion, 18, $38-50$.

Faltinsen, O. M. and Timokha, A. N. (2009). Sloshing. Cambridge University Press (Cambridge). 
Frandsen, J. B. (2005). Numerical predictions of tuned liquid tank structural systems. J. Fluids \& Structures, 20, 309-329.

Herczyński, A. and Weidman, P. D. (2012). Experiments on the periodic oscillation of free containers driven by liquid sloshing. J. Fluid Mech., 693, 216-242.

Ibrahim, R. A. (2005). Liquid Sloshing Dynamics. Cambridge University Press (Cambridge).

Ikeda, T. and Nakagawa, N. (1997). Non-linear vibrations of a structure caused by water sloshing in a rectangular tank. J. Sound Vibr., 201, 23-41.

Mayer, H. C. and Krechetnikov, R. (2012). Walking with coffee: why does it spill? Phys. Rev. E, 85, 046117.

Meyer, K. R. and Hall, G. R. (1992). Introduction to Hamiltonian Dynamical Systems and the $N$-Body Problem. Springer-Verlag (Berlin).

Moiseev, N. N. (1953). The problem of solid objects containing liquids with a free surface. Mat. Sbornik., 32(74)(1), 61-96 (in Russian).

Moiseyev, N. N. and Rumyantsev, V. V. (1968). Dynamic Stability of Bodies Containing Fluid. Springer-Verlag (New York).

Turner, M. R. and Bridges, T. J. (2013). Nonlinear energy transfer between fluid sloshing and vessel dynamics. J. Fluid Mech., 719, 606-636.

Weidman, P. D. (1994). Synchronous sloshing in free and suspended containers. APS Division of Fluid Dynamics, 47th Annual Meeting, Atlanta, GA 20-22 November.

Weidman, P. D. (2005). Sloshing in suspended containers. APS Division of Fluid Dynamics, 58th Annual Meeting, Chicago, IL 20-22 November.

Xue, S., Ko, J., and Xu, Y. (2000). Tuned liquid column damper for suppressing pitching motion of structures. Engineering Structures, 22(11), 1538-1551.

Yu, J. (2010). Effects of finite water depth on natural frequencies of suspended water tanks. Stud. Appl. Math., 125, 337-391. 